\title{
Dilute Solution Properties and Unperturbed Chain Dimension of Cellulose Triacetate
}

\author{
Kenji Kamide, Yukio MiYAZAKI, and Tatsuyuki ABE \\ Textile Research Laboratory, Asahi Chemical Industry Company, Ltd., \\ Takatsuki, Osaka 569, Japan.
}

(Received November 30, 1978)

\begin{abstract}
Cellulose triacetate(CTA, combined acetic acid content, $61.0 \mathrm{wt} \%$ ) was successfully fractionated on the basis of molecular weight by successive solutional fractionation using 1chloro-2,3-epoxypropane as the solvent and hexane as the nonsolvent. The fractions cover a very wide range of molecular weights, having comparatively narrow molecular weight distribution $\left(M_{w} / M_{n}=1.4, M_{w}=\right.$ the weight-average molecular weight and $M_{n}=$ the number-average molecular weight). To estimate molecular parameter viscosities, osmotic pressure, and light scattering measurements were carried out on twelve CTA fractions in various solvents. The following empirical relations were obtained for the limiting viscosity number $[\eta]$ and the radius of gyration $\left\langle S^{2}\right\rangle_{z}^{1 / 2}$ in dimethylacetamide (DMAc) at $25^{\circ} \mathrm{C}:[\eta]=2.64 \times 10^{-2} M_{w}^{0.75} \quad\left(\mathrm{~cm}^{3} \mathrm{~g}^{-1}\right),\left\langle S^{2}\right\rangle_{z}^{1 / 2}=0.463 \times$ $10^{-8} M_{w}{ }^{0.55}(\mathrm{~cm})$. For CTA/DMAc system, the CTA chain is gaussian in the unperturbed state and $a_{\Phi}(=\operatorname{dln} \Phi / \operatorname{dln} M, \Phi=$ Flory's viscosity parameter $)=0.106$ and the draining parameter $X=2$ were obtained. On the basis of the light scattering and viscosity data, the short-range interaction parameter $A=1.46 \times 10^{-8} \mathrm{~cm}$, the conformation parameter $\sigma=3.14$, and the characteristic ratio $C_{\infty}=20.4$. These values were estimated by using method $2 \mathrm{~B}, 2 \mathrm{C}$, and $2 \mathrm{G}$, proposed in the previous paper (This journal, 10, 409 (1978)). The $A$ value in polar solvents (group I: DMAc, trifluoroacetic acid, and acetone) is significantly larger than that in non-polar solvents (group II: dichloromethane, tetrachloroethane, and trichloromethane). The effect of the degree of substitution on $[\eta]$ and $\sigma$ for cellulose acetate in acetone is just the reverse of those for cellulose nitrate in the same solvent. The CTA chain is semiflexible and the contribution of the volume effect on $[\eta]$ is much smaller than that of the draining effect.
\end{abstract}

KEY WORDS Dilute Solution Property / Unperturbed Chain Dimension/ Cellulose Triacetate / Cellulose Diacetate / Viscosity / Osmotic Pressure / Light Scattering / Draining Effect / Conformation Parameter / Characteristic Ratio /

Cellulose triacetate (we denote it by CTA hereafter) has held for many years, worldwide importance in industry as a staring polymeric material for fibers, plastics, films, and membranes. Nevertheless, very few comprehensive and reliable studies on its molecular characteristics have been reported up to now. Only the solubility behaviour of CTA in various solvents and their Mark-Houwink-Sakurada (MHS) equations (eq 6) have been the targets for studies in the past. In addition, all MHS equations, proposed hitherto, are less accurate ${ }^{1}$ and are still not completely established. For instance, the Polymer Handbook compiles nine MHS equations for "CTA" solutions. ${ }^{2}$ It is particularly noteworthy, however, that all these equations except for the Flory et al.'s equation, are for cellulose diacetate (CDA) and not for CTA. More recently, during the course of our investigation, Nair et $a l^{3}$ carried out light scattering measurements on CTA fractions in a mixed solvent [dichloromethane(DCM)/ methanol $=1 / 1, \mathrm{v} / \mathrm{v}]$ and viscosity measurements in trichloromethane (TCM) over a very limited range of molecular weight (the ratio of the maximum to minimum weight-average molecular weight $M_{w}$, is only 1.72). As will be discussed later, their results are far beyond our understanding and in particular, the values of the radius of gyration $\left\langle S^{2}\right\rangle_{z}{ }^{1 / 2}$ are extraordinarily large. The main reason why the research of CTA dilute solution has been delayed and is still in a rather primitive state, although great advances have 
made in elucidation of the molecular parameters for other cellulose derivatives, should be attributed exclusively to the great difficulty encountered in experiment on molecular weight fractionation. In fact from among a large number of studies of the molecular weight fractionation of CTA published since the mid-1930's, we can find very few successful examples.

In our earlier work, ${ }^{4}$ we determined the molecular properties of cellulose diacetate (CDA, combined acetic acid content (AC), $55.6 \mathrm{wt} \%$ ) in solutions. In this work, we attempt to fractionate CTA efficiently by the solubility method and to measure, over a wide range of molecular weights, the dilute solution properties of CTA fractions, thus obtained, by viscometry, membrane osmometry (MO), and light scattering (LS) method and to determine in a general way the molecular parameters of CTA in comparison with CDA.

\section{EXPERIMENTAL}

\section{Polymer Sample and Solvents}

Purified cotton linter was acetylated by the standard method with an acetic anhydride-acetic acid-sulfuric acid mixture. ${ }^{5,6}$ The product was subjected to additional stabilization by an excess magnesium acetate. A proper amount of $35 \mathrm{wt} \%$ acetic acid was added to produce the precipitate completely which was filtered through filter paper and then washed with water. CTA, thus prepared (sample cord TA2), was dried in vacuo and had the numberaverage molecular weight $M_{n}=5.85 \times 10^{4}$ by $\mathrm{MO}$ and the weight-average molecular weight $M_{w}=2.35 \times 10^{5}$ by LS. The combined acetic acid content (AC) determination of the unfractionated CTA by the back-titration method using sodium hydroxide and sulfuric acid gave a value of $61.0 \mathrm{wt} \%$ (degree of substitution (DS) $=2.89$ ).

1-chloro-2,3-epoxypropane, hexane, trifluoroacetic acid (TFA), acetone, dichloromethane (CDM), 1,1,2,2-tetrahcloroethane (TCE), and trichloromethane (TCM) were of reagent grade and used as received. Dimethylacetamide (DMAc), used for light scattering method, was of guaranteed grade and was purified by drying over phosphorous pentaoxide and distillation immediately prior to use.

\section{Fractionation}

Up to now, acetic acid and chlorinated hy- drocarbons, which have the low dielectric constant $\varepsilon$, have been extensively employed as solvents for the molecular weight fractionation of CTA. The fractionation efficiency achieved by using the abovementioned solvents was poor unfortunately and the numerous attempts made so far have met with very limited success.

In order to overcome the above-mentioned experimental difficulty, preliminary experiments on phase separation of CTA solution were performed for many solvent/nonsolvent combinations, including those employed in literature ${ }^{3,7-21}$. Judging from the easiness of two liquid phase separation and of the solvent recovery, we chose 1-chloro-2,3epoxypropane (epichlorohydrine) as solvent and hexane as precipitant.

Successive solutional fractionation technique (SSF), originally advocated for use by Kamide and his coworkers, ${ }^{22}$ was applied. A 60 gram TA2 sample was dissolved in 1-chloro-2,3-epoxypropane $\left(6000 \mathrm{~cm}^{3}\right)$ and thermostated at $35^{\circ} \mathrm{C}$. The requisite amount of hexane predetermined by a pilot fractionation, was added to the solution, resulting in phase separation. The supernatant phase was isolated by a vacuum line from the vessel and hexane and 1chloro-2,3-epoxypropane in the phase were separated by step-wise evaporation in a rotary evaporator and re-used for further fractionation. The fractionation was carried out in a perfectly closed system. The fractionation appratus was specially designed and is described elsewhere. ${ }^{23}$ Finally, thirteen fractions were separated in a SSF run, in which the composition of the hexane at each step varied from 44.5 to 33.4 vol $\%$ at $35^{\circ} \mathrm{C}$. Equilibrium between the two phases was not difficult to attain, so that the fractionation is efficient (see Table I). The polymer fractions prepared in this way were vacuumdried at $60^{\circ} \mathrm{C}$ for one day. No hydrolysis of the acetyl group was detected.

\section{Solution Viscosity}

The CTA solutions for viscosity mesurement were prepared at room temperature, excluding DMAc, in which the polymer was dissolved at $60^{\circ} \mathrm{C}$. The CTA solution in acetone was prepared by the cooling method used in a previous paper. ${ }^{4}$ It has been known that CTA dissolves in acetone by cooling a slurry of CTA and acetone down to $-40^{\circ} \mathrm{C}$ or below, followed by warming it to room temperature. ${ }^{21,24} \mathrm{As}$ early as the late 1920 s, Kita et al. ${ }^{25}$ first pointed out 
that the acetone solubility of CTA is mainly attributable not to the acetyl content, but to the "physical state" of polymer. Solution viscosity of CTA fractions was measured in DMAc, TFA, acetone, TCE, and $\mathrm{TCM}$ at $25^{\circ} \mathrm{C}$ and in $\mathrm{DCM}$ at $20^{\circ} \mathrm{C}$, by using Ubbelohde-type capillary viscometers, specially designed so that no kinetic energy correction was necessary. The limiting viscosity number $[\eta]$, expressed in $\mathrm{cm}^{3} \mathrm{~g}^{-1}$, was determined as usual from Huggins plot $\left(\eta_{\mathrm{sp}} / c v s . c ; \eta_{\mathrm{sp}}\right.$, specific viscocisty and $c$, concentration). For the sake of comparison, $[\eta]$ of five CDA fractions prepared in the previous paper ${ }^{4}$ was measured in DMAc, TFA, and acetone at $25^{\circ} \mathrm{C}$.

\section{Membrane Osmometry}

Osmotic pressure measurements were carried out with a Howlett Packard high speed membrane osmometer model 502 in DMAc, acetone, TCE, and $\mathrm{TCM}$ at $25^{\circ} \mathrm{C}$. The membrane was a Sartorious allerfeinst membrane and no diffusing of the solute was observed.

The following relation holds between the osmotic pressure $\pi$ and the concentration $c\left(\mathrm{~g} \mathrm{~cm}^{-3}\right)$

$$
\pi / c=R T\left\{1 / M_{n}+A_{2, o} c+\cdots\right\}
$$

where $R$ is the gas constant, $T$ is temperature $(\mathrm{K})$ and $A_{2, O}$ is the second virial coefficient obtained by membrane osmometry. $M_{n}$ and $A_{2, O}$ were evaluated from the linear part of a plot of $\pi / c v s . c$ as intercept and slope, respectively.

The polymer-solvent thermodynamic interaction parameter $\chi$ was calculated from the osmotic data using the relation

$$
\chi=-\left\{\pi V_{0} / R T+\ln \left(1-v_{\mathrm{p}}\right)+\left(1-1 / X_{n}^{*}\right) v_{\mathrm{p}}\right\} / v_{\mathrm{p}}^{2}
$$

where $V_{0}$, solvent molar volume; $v_{\mathrm{p}}$, volume fraction of polymer $\left(=V_{0} c / M\right) ; X_{n}{ }^{*}$, the number-average molar volume ratio of polymer to solvent $\left(=M_{n}\right.$ $V_{\mathrm{m}} / m ; V_{\mathrm{m}}$, molar volume of the monomer unit and $m$, molecular weight of the monomer unit).

\section{Light Scattering}

In preference to acetone DMAc was chosen as the solvent for the light scattering measurement, since it affords easy exclusion of the gel-like materials in solution. Two vacuum-dried CTA fractions (TA2-6 and TA2-13) and a CDA fraction (EF 3-11), obtained in the previous work, ${ }^{4}$ were dissolved in freshly distilled DMAc at $60^{\circ} \mathrm{C}$. The specific re- fractive index increment $\mathrm{d} n / \mathrm{d} c$ of these solutions was determined in a Shimadzu differential refractiometer model DR-4 at $25^{\circ} \mathrm{C}$ at a wave length $\lambda_{0}$ of $436 \mathrm{~nm}$ $(4360 \AA)$. Great care is required to ensure that DMAc is absolutely dry, since it is highly hygroscopic and will yield a high $\mathrm{d} n / \mathrm{d} c$ value when wet. The $\mathrm{d} n / \mathrm{d} c$ was found to be $0.0398\left(\mathrm{~cm}^{3} \mathrm{~g}^{-1}\right)$ for two CTA fractions, irrespective of their $M_{w}$ values, and $0.0418\left(\mathrm{~cm}^{3} \mathrm{~g}^{-1}\right)$ for a CDA $(\mathrm{AC}=55.6 \mathrm{wt} \%)$ fraction.

The optical clarification procedure of the solution is similar to that used in the previous paper. ${ }^{4}$ After a $0.5 \%$ stock solution was centrifuged at $3 \times 10^{4} \mathrm{~g}$ for $90 \mathrm{~min}$, the fresh solvent was added to the isolated upper layer, yielding the solutions with four different concentrations. The solutions prepared thus far were immediately followed again by centrifugation at $3 \times 10^{4} \mathrm{~g}$ for $90 \mathrm{~min}$. The upper two-thirds of the supernatant phase was carefully filtered through a Sartorious membrane filter $0.2 \mu \mathrm{m}$, directly into a light scattering cylindrical cell.

The light scattering measurements in the angle range $30-150^{\circ}$ were made with veritcally polarized incident light $\left.\left[\lambda_{0}\right]=436 \mathrm{~nm}(4360 \AA)\right]$ at $25^{\circ} \mathrm{C}$ in the Shimadzu-Brice type light scattering photometer model PG-21. The vertical component of the scattered light was measured. $M_{w}$ and $A_{2,1}$ (the suffix 1 means light scattering) were determined from the Zimm plot. No distortion was observed in the Zimm plot for CTA solutions after ultracentrifugation for removing the gel particles attributable to non-CTA materials and in part to the associated CTA molecules.

\section{RESULTS AND DISCUSSION}

\section{Molecular Weight and Molecular Weight Distribution of CTA Fraction}

Table I gives a list of the experimental results by the membrane osmometry and light scattering for CTA solutions in DMAc, all evaluated at $25^{\circ} \mathrm{C}$. In Table II are outlined the viscosity data obtained with solutions of the same CTA fractions in DMAc, TFA, acetone, DCM, TCE, and TCM. All measurements were made, with the exception of DCM, at $25^{\circ} \mathrm{C}$. $\left\langle S^{2}\right\rangle_{z}{ }^{1 / 2}$ and $\left\langle S^{2}\right\rangle_{w}{ }^{1 / 2}$, tabulated in the fifth and sixth columns, represent the $Z$-average and weightaverage radius of gyration, respectively. The $\left\langle S^{2}\right\rangle_{w}{ }^{1 / 2}$ values were converted from $\left\langle S^{2}\right\rangle_{z}{ }^{1 / 2}$ and the polydispersity $\left(M_{w} / M_{n}\right)$ data through use of the 
K. Kamide, Y. MiYazaKi, and T. AbE

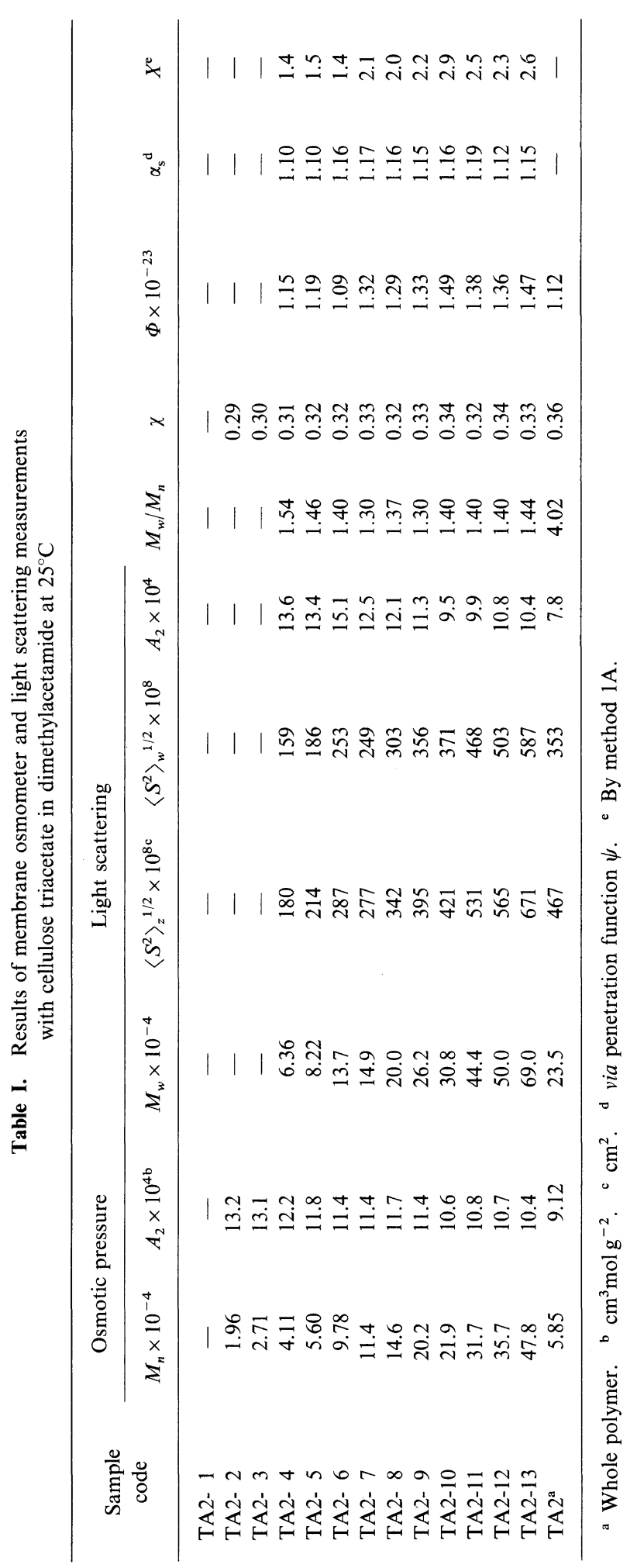


Dilute Solution Properties of Cellulose Triacetate

Table II. Results of viscosity measurements with cellulose triacetate in various solvents

\begin{tabular}{|c|c|c|c|c|c|c|c|}
\hline \multirow{2}{*}{$\begin{array}{l}\text { Sample } \\
\text { code }\end{array}$} & \multirow{2}{*}{$M_{w} \times 10^{-4}$} & \multicolumn{6}{|c|}{$\mathrm{cm}^{3} \mathrm{~g}^{-1}\left(\right.$ at $\left.25^{\circ} \mathrm{C}\right) /[\eta]$} \\
\hline & & $\mathrm{DMAc}^{\mathrm{a}}$ & $\mathrm{TFA}^{\mathrm{b}}$ & Acetone & $\mathrm{DCM}^{\mathrm{c} \cdot \mathrm{d}}$ & $\mathrm{TCE}^{\mathrm{e}}$ & $\mathrm{TCM}^{\mathrm{f}}$ \\
\hline TA2- 1 & - & - & - & - & - & 26 & - \\
\hline TA2- 2 & - & 59 & - & - & - & 38 & - \\
\hline TA2- 3 & - & 70 & - & - & - & 47 & - \\
\hline TA2- 4 & 6.36 & 102 & 96 & - & 59 & 60 & $(47)^{\mathrm{g}}$ \\
\hline TA2- 5 & 8.22 & 131 & 118 & 110 & 71 & 73 & 69 \\
\hline TA2- 6 & 13.7 & 183 & 164 & 143 & 100 & 95 & 97 \\
\hline TA2- 7 & 14.9 & 195 & - & 154 & - & 98 & - \\
\hline TA2- 8 & 20.0 & 256 & 228 & 211 & 136 & 129 & 129 \\
\hline TA2- 9 & 26.2 & 327 & - & 265 & - & 163 & - \\
\hline TA2-10 & 30.8 & 350 & 286 & 282 & 184 & 168 & 171 \\
\hline TA2-11 & 44.4 & 451 & 385 & 357 & - & 214 & 210 \\
\hline TA2-12 & 50.0 & 490 & 434 & 398 & 260 & 243 & 228 \\
\hline TA2-13 & 69.0 & 607 & 508 & 480 & 306 & 270 & 272 \\
\hline
\end{tabular}

Table III. Results of osmotic pressure measurements with cellulose triacetate fractions in various solvents at $25^{\circ} \mathrm{C}$

\begin{tabular}{|c|c|c|c|c|c|c|c|c|c|c|c|c|}
\hline \multirow{2}{*}{$\begin{array}{l}\text { Sample } \\
\text { code }\end{array}$} & \multicolumn{4}{|c|}{$M_{n} \times 10^{-1}$} & \multicolumn{4}{|c|}{$A_{2} \times 10^{4} / \mathrm{mol} \mathrm{cm}^{3} \mathrm{~g}^{-1}$} & \multicolumn{4}{|c|}{$\chi$ from eq 2} \\
\hline & $\mathrm{DMAc}^{\mathrm{a}}$ & Acetone & $\mathrm{TCE}^{\mathrm{b}}$ & $\mathrm{TCM}^{\mathrm{c}}$ & $\mathrm{DMAc}^{\mathrm{a}}$ & Acetone & $\mathrm{TCE}^{\mathrm{b}}$ & $\mathrm{TCM}^{\mathrm{c}}$ & $\mathrm{DMAc}^{\mathrm{a}}$ & Acetone & $\mathrm{TCE}^{\mathrm{b}}$ & $\mathrm{TCM}^{\mathrm{c}}$ \\
\hline TA2- 5 & 5.60 & 5.45 & 5.80 & 5.60 & 11.8 & 11.7 & 12.0 & 12.4 & 0.32 & 0.36 & 0.29 & 0.33 \\
\hline TA2-10 & 21.9 & 21.1 & 21.2 & 21.7 & 10.6 & 11.7 & 11.5 & 8.7 & 0.34 & 0.36 & 0.30 & 0.38 \\
\hline
\end{tabular}

a Dimethylacetamide. ${ }^{\mathrm{b}}$ Tetrachloroethane. ${ }^{\mathrm{c}}$ Trichloromethane.

relation

$$
\left\langle S^{2}\right\rangle_{w}^{1 / 2}=\left(\frac{h+1}{h+2}\right)^{1 / 2}\left\langle S^{2}\right\rangle_{z}^{1 / 2}
$$

with

$$
h^{-1}=\left(M_{w} / M_{n}\right)-1
$$

Equation 3 was derived for the case when the molecular weight distribution (MWD) of the polymer sample was represented by the SchulzZimm type distribution.

Flory's viscosity parameter $\Phi$ was calculated directly from eq 5 using the experimentally determined values

$$
\Phi=[\eta] M_{w} /\left\langle S^{2}\right\rangle_{z}^{3 / 2} 6^{3 / 2} q_{w, z}
$$

with $q_{w, z}=$ the correction factor for the polymolecularity of the sample. The $\chi$ value, calculated by eq 2 , showed no significant concentration dependence within the concentration range studied; averaged values over the concentration covered here are shown in the Table I.

The CTA fractions separated in this work cover a wide molecular weight $\left(M_{w}\right)$ range from $2.9 \times 10^{4}$ to $69.0 \times 10^{4}$ (i.e., the ratio of the upper- to the lowerlimited $M_{w}=23.8$ ). The polydispersity, expressed in terms of $M_{w} / M_{n}$, of the CTA fractions lies between $1.30-1.54$ and there is a reasonably constant $M_{w} / M_{n}$ ratio of about 1.4 over the $M_{w}$ range. Theoretical calculations ${ }^{26-29}$ as well as the actual experiments on polyethylene, ${ }^{23}$ polystyrene, ${ }^{30-33}$ poly $\left(\alpha\right.$-methyl styrene) ${ }^{34}$ and CDA,${ }^{4}$ carried out at our laboratory, show that SSF always yields a series of fractions with nearly the same $M_{w} / M_{n}$, except for a few initial fractions. The results on CTA are in an excellent agreement with previous conclusions we reached, and these data do not seem to be the possibility of much further advance in preparing 
CTA having very narrow MWD $\left(M_{w} / M_{n} \simeq 1.1\right)$, without finding of a new single solvent suitable for SSF. Molecules of cellulose derivatives have a peculiar tendency to associate in poor solvents. CDA in TCE below $70^{\circ} \mathrm{C}$ is just an example. ${ }^{4}$ For the purpose of clarifying this point to the case of CTA solutions in the solvents employed here, osmotic pressures of solutions of two CTA fractions, differing $M_{w}$ (TA2-5 and TA2-10), in various solvents were measured. The results of these determinations are represented in Table III. Evidently, four solvents gave almost the same $M_{n}$ value of CTA fraction, within the experimental error. This indicates directly that molecular association of CTA dose not occur in these solvents. As early as 1953, Staudinger and Eicher ${ }^{35}$ found by the membrane osmometry a fairly good coincidence of $M_{n}$ of a CDA (DS $=2.37$ ) sample in acetone with that in glacial acetic acid. After the measurement of $M_{n}$ for a CTA (DS $=2.88$ ) whole polymer in nitromethane, TCM, and acetone, Cowie and Ranson ${ }^{21}$ concluded that neither degradation nor aggregation was apparent.

It has been generally believed that CTA is soluble only in acidic solvents. ${ }^{36}$ But non-acidic solvents, such as DMAc, TFA, and acetone are also adequate solvents for CTA.

\section{Mark-Houwink-Sakurada Equation}

Figure 1a shows the log-log plot of $[\eta] v s . M_{n}$ or $M_{w}$ for CTA solution in DMAc at $25^{\circ} \mathrm{C}$. In this figure, the closed circle denotes $M_{n}$ and the open circle is $M_{w}$. Both plots can be reasonably represented by straight lines, suggesting that the Mark-Houwink-Sakurada equation,

$$
[\eta]=K_{\mathrm{m}} M^{a}
$$

or

$$
[\eta]=K_{\mathrm{m}} M_{w}^{a}
$$

may be well established over the entire $M_{n}$ range investigated.

The parameters $K_{\mathrm{m}}$ and $a$ of MHS equations for six solvents were evaluated by using the least square method and MHS equations are represented, in Figure $1 \mathrm{~b}-\mathrm{f}$, by straight lines through the observed points. MHS equations of this work are the most comprehensive correlations so far made between $[\eta]$ and the molecular weight. In general, $[\eta]$ values in CTA solution in chlorinated hydrocarbons are smaller than those of the same molecular weight in other

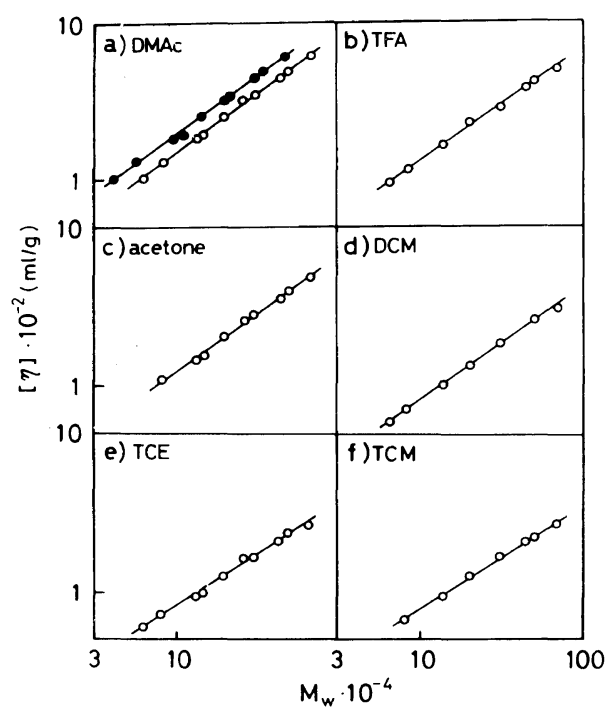

Figure 1. Log-log plot of limiting viscosity number $[\eta]$ against the weight-average molecular weight $M_{w}$ (open mark) or the number-average molecular weight $M_{n}$ (closed mark) for cellulose triacetate in dimethylacetamide (DMAc), trifluoroacetic acid (TFA), acetone, dichloromethane (DCM), tetrachloroethane (TCE), and trichloromethane (TCM). Straight lines represent the Mark-Houwink-Sakurada equations (eq a-g) determined by the least-squares method.

solvents by $50 \%$.

The MHS equation corresponding to monodisperse CTA in DMAc can be readily derived from $[\eta]$ and $M_{w}$ of CTA fractions, by taking into account the MWD of the fractions, as

$$
[\eta]=2.71 \times 10^{-2} M^{0.750}
$$

In deriving eq a we assumd the Schulz-Zimm distribution with $M_{w} / M_{n}=1.4$ for the samples. Almost the same equation as eq a can also be derived from $[\eta]$ and $M_{n}$ of the fractions.

Table IV lists the MHS equations for CTA established in this work, together with those proposed hitherto. The exponent $a$ values reported in the literature data for the systems studied here are generally in the range of $0.8 \sim 1.0$, and are indeed markedly larger than our values $(0.65 \sim 0.75)$. Notable differences in parameters in the MHS equations, as shown, are considered to have their origin in the considerable molecular weight dependence of the polydispersity of the samples used in literature, as demonstrated with CDA solutions. ${ }^{4}$ In particular, the systematic molecular weight depen- 
Dilute Solution Properties of Cellulose Triacetate

Table IV. Mark-Houwink-Sakurada equations for cellulose triacetate in various solvents

\begin{tabular}{|c|c|c|c|c|c|c|c|c|c|}
\hline \multirow{2}{*}{ Solvent } & \multirow{2}{*}{$\frac{\text { Temp }}{{ }^{\circ} \mathrm{C}}$} & \multirow{2}{*}{$K_{\mathrm{m}} \times 10^{2}$} & \multirow{2}{*}{$a$} & \multicolumn{2}{|c|}{ No. of sample } & \multirow{2}{*}{$\begin{array}{c}\text { Mol wt range } \\
M \times 10^{-4}\end{array}$} & \multirow{2}{*}{ Method } & \multirow{2}{*}{$\begin{array}{c}\begin{array}{c}\text { Acetyl } \\
\text { content }\end{array} \\
\frac{\mathrm{o}}{\mathrm{o}}\end{array}$} & \multirow{2}{*}{ Ref } \\
\hline & & & & Fr. & W.P. & & & & \\
\hline \multirow{2}{*}{$\begin{array}{l}\mathrm{DCM}^{\mathrm{a}} / \\
\text { ethanol } \\
(8 / 2, \mathrm{v} / \mathrm{v})\end{array}$} & 25 & 1.41 & 0.834 & 24 & - & $2.15-20.4$ & $\operatorname{vis}^{\mathrm{f}}$ & 60.9 & 14 \\
\hline & 25 & 0.45 & 0.90 & - & 5 & $3.06-18.0$ & vis ${ }^{g}$ & 62 & 38,39 \\
\hline \multirow[t]{3}{*}{$\mathrm{TCM}^{\mathrm{b}}$} & 25 & 2.51 & 1.02 & - & 8 & & $\mathrm{MO}^{\mathrm{h}}$ & 61.0 & 40 \\
\hline & 25 & 4.54 & 0.649 & 7 & - & $8.22-69.0$ & $\mathrm{LS}^{\mathrm{i}}$ & 61.0 & this work \\
\hline & 20 & 0.22 & 0.95 & 13 & - & $1.36-13.0$ & MP & 61 & 18 \\
\hline DCM & 20 & 2.47 & 0.704 & 7 & - & $6.36-69.0$ & $\mathrm{LS}$ & 61.0 & \\
\hline$D M A c^{c}$ & 25 & 2.64 & 0.750 & 10 & - & $6.36-69.0$ & LS & 61.0 & \\
\hline TFA $^{d}$ & 25 & 3.96 & 0.706 & 8 & - & $6.36-69.0$ & LS & 61.0 & this work \\
\hline Acetone & 25 & 2.89 & 0.725 & 9 & - & $8.22-69.0$ & LS & 61.0 & \\
\hline $\mathrm{TCE}^{\mathrm{e}}$ & 25 & 3.93 & 0.662 & 10 & - & $6.36-69.0$ & LS & 61.0 & \\
\hline
\end{tabular}

${ }^{a}$ Dichloromethane. ${ }^{b}$ Trichloromethane. ${ }^{\mathrm{c}}$ Dimethylacetamide. ${ }^{\mathrm{d}}$ Trifluoroacetic acid. ${ }^{\mathrm{e}}$ Tetrachloroethane. ${ }^{f}$ Degree of acetylation was calculated from the cuprammonium limiting viscosity number of diacetylated fractions: $[\eta]=0.0319 \times P_{n}{ }^{0.657}\left(P_{n}\right.$, the number-average degree of polymerization was determined for CDA by osmometry). ${ }_{\mathrm{g}} M$ was calculated from the relationships for cellulose diacetate in acetone at $25^{\circ} \mathrm{C}:[\eta]=8.97 \times 10^{-3} M_{n}{ }^{0.90}$, which is given by Phillips and Bjork. ${ }^{39} \mathrm{~h}$ Membrane osmometry. ${ }^{\mathrm{i}}$ Light scattering.

dence of MWD of the samples will yield incorrectly high $a$ values. Detailed discussions of the effect of MWD on MHS equation are presented eleswhere. ${ }^{37}$

\section{Radius of Gyration and Second Virial Coefficient}

Figure 2 shows the radius of gyration $\left\langle S^{2}\right\rangle_{z}{ }^{1 / 2}$ plotted against the molecular weight for CTA solution in DMAc as open circle. From this figure, we obtain the following empirical relation.

$$
\left\langle S^{2}\right\rangle_{z}{ }^{1 / 2}=0.463 \times 10^{-8} M_{w}{ }^{0.55}
$$

In Figure 2 are also included the data from Nair et al.'s work ${ }^{3}$ on - CTA in a mixted solvent $(\mathrm{DCM} /$ methanol, $1 / 1, \mathrm{v} / \mathrm{v})$ as a closed circle, together with the point by Shakhparonov et al. ${ }^{41}$ on CTA in DCM (temperature was not described) as a closed rectangle. The Nair et al.'s value of $\left\langle S^{2}\right\rangle_{z}{ }^{1 / 2}$ is, indeed, larger by a factor of about 3.5 than the value obtained here for DMAc, compared at the same $M_{w}$. Thus, it is obvious that Nair et al.'s data of $\left\langle S^{2}\right\rangle_{z}{ }^{1 / 2}$ are the largest among all data available for cellulose derivatives. ${ }^{42}$ The data points obtained by Shakhparonov et al.$^{41}$ for CTA in TCM is slightly smaller than those we obtained in DMAc. It is expected from the $[\eta]$ data in Table II that $\left\langle S^{2}\right\rangle_{z}{ }^{1 / 2}$ is smaller in TCM than in DMAc. An open triangle in the figure corresponds to CDA in DMAc (see Table VI).

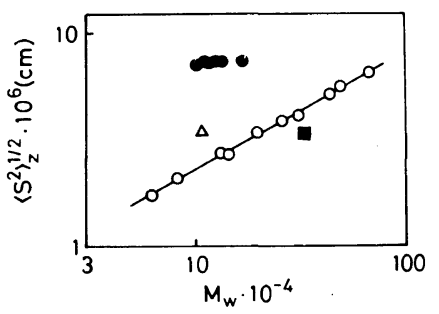

Figure 2. Molecular weight dependence of the radii of gyration $\left\langle S^{2}\right\rangle_{z}{ }^{1 / 2}$ for cellulose triacetate (CTA) and cellulose diacetate (CDA) solutions: $O$, CTA in dimethylacetamide (DMAc) (this work); , CTA in dichloromethane (DCM)/methanol (1/1, v/v) (Nair et al.'s work $^{3}$ ); $\mathbf{\square}$, CTA in DCM (Shakhparonov et al.'s work ${ }^{41}$; $\triangle$, CDA in DMAc (this work).

Figure 3 shows the log-log plot of the second virial coefficient $A_{2}$ against $M_{w}$, for CTA solution in DMAc at $25^{\circ} \mathrm{C} . A_{2}$ shows a small molecular weight dependence. In the lower $M_{w}$ range, $A_{2, l}$ is slightly larger than $A_{2, o}$. However, if we take into consideration the effect of MWD on $A_{2}$ and the experimental error involved, there is essentially no difference between $A_{2, l}$ and $A_{2, o}$. The polydispersity correction of the CTA samples, according to Casassa, ${ }^{43}$ reduces the observed $A_{2, l}$ only by about $1 \%$ (and $A_{2,0}$ by about $9 \%$ ). The second virial coefficient by membrane osmometry $A_{2, O}$ is related experimentally to its $M_{w}$ through the equation: 


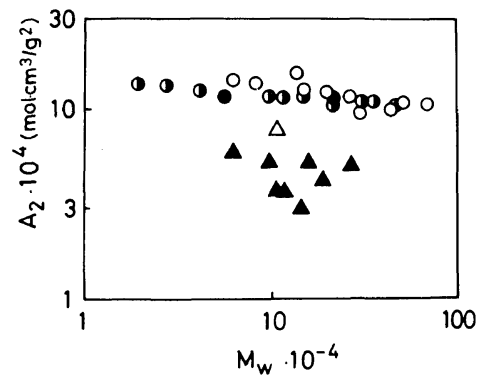

Figure 3. Molecular weight dependence of the second virial coefficient $A_{2}$ for cellulose triacetate (CTA) and cellulose diacetate (CDA) solutions: $\bigcirc$, CTA in dimethylacetamide (DMAc) by light scattering (LS); 1 , CTA in DMAc by membrane osmometry (MO); CTA in acetone by $\mathrm{MO} ; \triangle, \mathrm{CDA}$ in DMAc by LS; $\boldsymbol{\Delta}, \mathrm{CDA}$ in acetone by $\mathrm{LS}$.

$$
A_{2, O}=2.64 \times 10^{-3} M_{w}^{-0.071}
$$

for a CTA solution in DMAc at $25^{\circ} \mathrm{C}$. In Figure 3, the corresponding data for CDA solutions in DMAc and acetone are plotted for the sake of comparison. The $A_{2}$ values for CTA in DMAc are more than two times the values for CDA in acetone.

The $A_{2, o}$ values of CTA in DMAc and acetone are similar in magnitude, and slightly larger than those in the chlorinated solvents. The polymer-solvent interaction parameter $\chi$ for CTA is in the range $0.29-0.38$ in DMAc, acetone, TCE, and TCM (Tables I and III). Howard and Parikh obtained $\chi=0.38$ for an unfractionated CTA sample $\left(M_{n}=3.82 \times 10^{4}\right)$ solution in TCM. $^{19}$ This value agrees fairly well, if the MWD of the sample is taken into account with those evaluated here. There is no obvious relation between $[\eta]$ and $\chi$ (or $A_{2}$ ) of a given sample dissolved in different solvents.

\section{Flory's Viscosity Parameter and Excluded Volume Effect}

Figure 4a illustrates the Flory's viscosity parameter $\Phi$ plotted as a function of the molecular weight for CTA solution in DMAc as open circle. As noted previously for other cellulose derivatives, the $\Phi$ value for CTA in DMAc reveals unquestionably a significant molecular weight dependence, which may be semiempirically expressed by

$$
\Phi=K_{\Phi} M_{\Phi}^{a}
$$

with $K_{\Phi}$ and $a_{\Phi}$ the parameters for a given polymer/solvent system. For CTA in DMAc, we

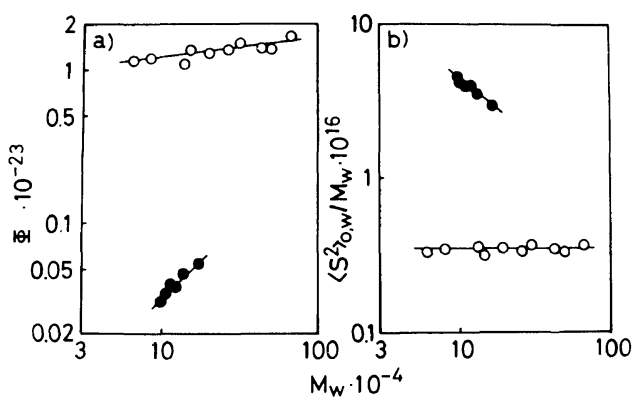

Figure 4. Molecular weight dependence of the Flory's viscosity parameter $\Phi$ (a) and of the ratio of the unperturbed radius of gyration $\left\langle S^{2}\right\rangle_{O, w} / M_{w}$ to $M_{w}$ for cellulose triacetate solutions (b): $\bigcirc$, CTA in dimethylacetamide (this work); , CTA in dichloromethane/methanol (1/1, v/v) (Nair et al.'s work ${ }^{3}$ ).

obtain $K_{\Phi}=0.35 \times 10^{23}$ and $a_{\Phi}=0.106$. In addition, the magnitude of $\Phi$ is less than $1.8-2.6$ times the theoretical value $\left(2.87 \times 10^{23}\right)$ at the unperturbed state. These experimental facts show definitely that the draining effect may by no means be ignored, and that it plays an important role in the CTA/DMAc system at least over the entire molecular weight range in the experiment. The significant contribution of the draining effect has not really attracted very much attention, until similar results were reported very recently for $\mathrm{CDA}^{4}$ and other cellulose derivatives. ${ }^{42}$ It is of interest to note that the $a_{\Phi}$ value of CDA in acetone is known to be exceptionally large. ${ }^{4}$

According to the Kurata-Yamakawa (KY) theory, ${ }^{44} \Phi$ is a function of the draining parameter $X$ in the form

$$
\Phi=\Phi(X) \alpha_{\mathrm{s}}{ }^{-(3-n(X))}
$$

where $\Phi(X)$ and $n(X)$ are functions of $X$, defined by KY theory. ${ }^{44}$ Therefore, on the basis of eq 5 and $5^{\prime}$ we can estimate $X$ values from the data of $[\eta], M_{w}$, $\left\langle S^{2}\right\rangle_{z}$ and the expansion factor $\alpha_{\mathrm{s}}$, which was calculated from the penetration function $\psi$, to be described later. This method was referred to as the method $1 \mathrm{~A}$ in the previous paper. ${ }^{42}$ The $X$ values thus estimated are brought together in the last column of Table I, and from which the averaged $X$ value was found to be 2.1 .

To check the reliability of the values of $\Phi$ and $a_{\Phi}$, which are very closely connected both with the draing parameter, the $X$ value was also evaluated from the following equations 


$$
a_{\Phi}=\frac{1}{2} \frac{\mathrm{d} \ln \Phi(X)}{\mathrm{d} \ln X}-\{3-n(X)\} a_{1} / 3
$$

and

$$
a_{1}=a-0.5-a_{\Phi}-1.5 a_{2}
$$

where $n(X)$ is defined in the $\mathrm{KY}$ theory and $a_{2}$ is a parameter representing the non-gaussian nature of the chain in the unperturbed state, and is defined by $\operatorname{d} \ln \left(\left\langle S^{2}\right\rangle_{0} / M\right) / \mathrm{d} \ln M\left(\left\langle S^{2}\right\rangle_{0}{ }^{1 / 2}\right.$, the radius of gyration at unperturbed state). On substituting numerical values for the quantities $a, a_{\Phi}$, and $a_{1}$, obtained for CTA/DMAc system (i.e., $a=0.750, a_{\Phi}=0.106$ and $a_{2}=0$ (see Figure $4 \mathrm{~b}$ ), into eq 8 and $9, X$ is found to be approximately 4 . Therefore, the values of $\Phi$ and $a_{\Phi}$ are roughly concordant, although the values determined by method $1 \mathrm{~A}$ are, beyond question, much more reliable.

The $\Phi$ values, calculated by using Nair et al.'s data, are represented in Figure 4a as closed circles. These values are, suprisingly, less than 10 times those we obtained and there are not examples of polymers, including cellulose derivatives/solvent system, showing such a small $\Phi$ values (for example, see Figure 5 of ref 42). Comparison of Figures 2 and 4, therefore, leads to the conclusion that Nair et al.'s data are not accurate enough to be analyzed, further for establishing empirically the dependence of $\Phi$ on $M_{w}$. Nair et al. estimated the short-range interaction parameter $A\left(=\left(6\left\langle S^{2}\right\rangle_{0} / M\right)^{1 / 2}\right.$ according to the Stockmayer and Fisman plot (by our notation, method $2 \mathrm{E}$ ), which retains its validity only in the case where both $a_{\Phi}$ and $a_{2}$ are concurrently zero.

The excluded volume parameter $\bar{z}$ was estimated from the penetration function $\psi$, defined as $0.746 \times 10^{-25} A_{2} M_{w}{ }^{2} /\left\langle S^{2}\right\rangle_{w}{ }^{3 / 2}$ ), and was calculated from the light scattering data in Table I, by the Kurata-Fukatsu-Sotobayashi-Yamakawa (KFSYII) equation, ${ }^{45}$

$$
\psi=\bar{z} h_{0}(\bar{z})=\frac{1-(1+3.903 \bar{z})^{-0.468}}{1.828}
$$

For the CTA/DMAc system, KFSY $-\mathrm{I}^{45}$ equation is not applicable, since the $\psi$ values for this system frequently exceed the upper limit of $\mathrm{KFSY}-\mathrm{I}^{45}$ equation (0.198). The values of the linear expansion factor $\alpha_{s}$ given in Table I were calculated unamibiguously from $\bar{z}$ according to the procedure already described, ${ }^{4,42}$ using the relation

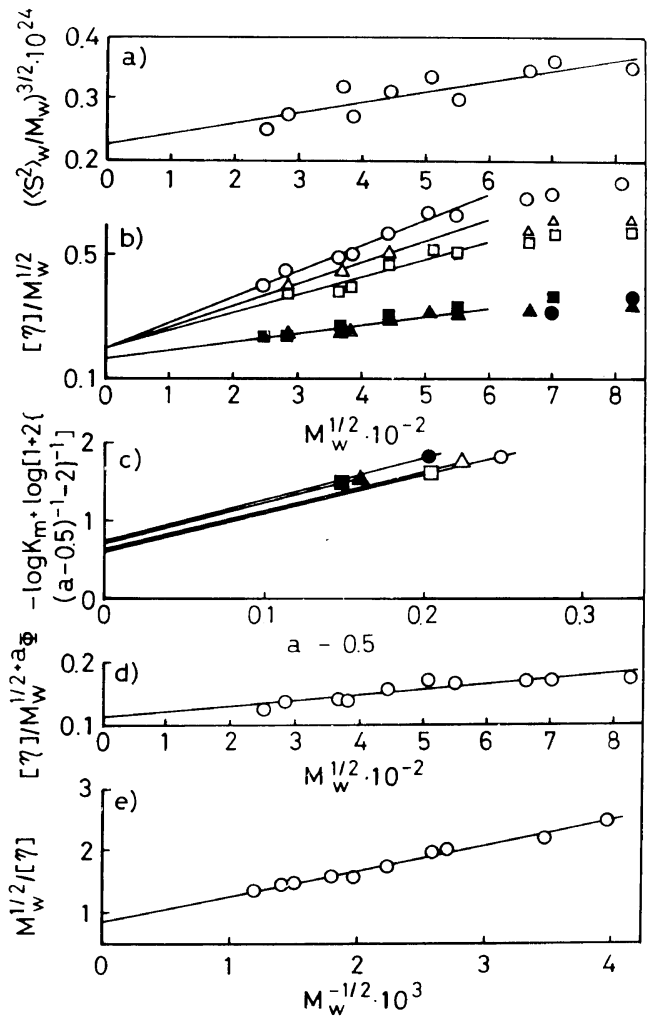

Figure 5. Baumann plot (a), Stockmayer-Fixman plot (b), Kamide et al. plot (c), Kamide-Miyazaki (I) plot (d) and Tanner-Berry plot (e) for cellulose triacetate in dimethylacetamide $(\bigcirc)$, trifluoroacetic acid $(\triangle)$, acetone $(\square)$, dichloromethane ( $\bullet$ ), tetrachloroethane $(\boldsymbol{\square})$, and trichloromethane $(\mathbf{A})$.

$$
\alpha_{\mathrm{s}}=(1-1.78 \bar{z})^{-1 / 3}
$$

For CTA/DMAc system, $\alpha_{\mathrm{s}} \leq 1.2$ was obtained and the excluded volume effect of this system is, as with other cellulose derivatives, small even at very high values of $M_{w}$.

\section{Unperturbed Chain Dimension}

The unperturbed chain dimension, $A$ ( $\equiv\left(\left\langle R^{2}\right\rangle_{0} / M\right)^{1 / 2},\left\langle R^{2}\right\rangle_{0}$, the mean-square end-toend distance in the unperturbed state) was estimated by using methods $2 \mathrm{~B}, 2 \mathrm{C}, 2 \mathrm{E}, 2 \mathrm{~F}$, and $2 \mathrm{H}$, proposed in the previous paper, ${ }^{42}$ for which the basic equations are as follows.

Method 2B:

$$
\left\langle S^{2}\right\rangle_{0}{ }^{1 / 2}=\left\langle S^{2}\right\rangle^{1 / 2} / \alpha_{\mathrm{s}}
$$


Method 2C:

$$
\left\langle S^{2}\right\rangle^{3 / 2} / M^{3 / 2}=A^{3 / 2} / 6^{3 / 2}+\left(1 / 4 \pi^{3 / 2}\right) B M^{1 / 2}
$$

(where $B$ is long range interaction parameter.) Method 2E:

$$
[\eta] / M^{1 / 2}=K+2(3 / 2 \pi)^{3 / 2} \Phi_{0}(\infty) B M^{1 / 2}
$$

(where $K=\Phi_{0}(\infty) A^{3}, \Phi_{0}(\infty)=2.87 \times 10^{23}$.)

Method 2F:

$$
\begin{gathered}
-\log K_{\mathrm{m}}+\log \left(1+2\left((a-0.5)^{-1}-2\right)^{-1}\right) \\
=-\log K+(a-0.5) \log M_{0}
\end{gathered}
$$

(where $M_{0}$ is a parameter depending on the molecular weight rang, $M_{1}-M_{2}$, and to which the MHS equation applies.)

Method 2G:

$$
\begin{gathered}
{[\eta] / M^{0.5+a_{\Phi}+1.5 a_{2}}} \\
=6^{3 / 2} K_{\Phi} K_{0}^{3 / 2}+0.66 K_{\Phi} B M^{\left(1-3 a_{2}\right) / 2}
\end{gathered}
$$

(where $\left.K_{0}=\left(\left\langle S^{2}\right\rangle_{0} / M\right) / M^{a}{ }_{2}\right)$

$$
M^{1 / 2} /[\eta]=\left(K^{\prime}\left(6\left\langle S^{2}\right\rangle / M\right)^{3 / 2}\right)^{-1}\left(1+A^{\prime} M^{-1 / 2}\right)(17)
$$

(where $K^{\prime}$ and $A^{\prime}$ are parameters depending on the models used.)

Values of $\left\langle S^{2}\right\rangle_{z}{ }^{1 / 2}$ in Table I were converted to values of $\left\langle S^{2}\right\rangle_{0, w}\left(=\left\langle S^{2}\right\rangle_{w} / \alpha_{\mathrm{s}}{ }^{2}\right)$ using these $\alpha_{\mathrm{s}}$ values (method 2B). The ratio $\left\langle S^{2}\right\rangle_{0 . w} / M_{w}$. plotted in Figure $4 \mathrm{~b}$ as a function of molecular weight, as an open mark for CTA solution in DMAc, is almost independent of $M_{w}$ and accordingly, the CTA chain can be reasonably treated as a gaussian chain, i.e., $a_{2}=0$, and the same applies for the CDA solution in THF $^{4}$. The closed mark in Figure $4 \mathrm{~b}$ is the $\left\langle S^{2}\right\rangle_{0 . w} / M_{w}$ data point, calculated for a CTA/a mixture of DCM and methanol from Nair et al.'s work $^{3}$. In conclusion, for the CTA/DMAc system we obtain $a_{\Phi}=0.106, a_{2}=0$ and $a_{1}\left(=a-0.5-a_{\Phi}\right.$ $\left.-1.5 a_{2}\right)=0.144$. This latter value agrees well with an $a$ value of 0.15 as calculated from eq $\mathrm{b}$ and $a_{2}=0$ by

$$
a_{1}=\frac{3}{2}\left\{\left(\mathrm{~d} \ln \left(\left\langle S^{2}\right\rangle_{w} / M\right) / \mathrm{d} \ln M\right)-a_{2}\right\}
$$

For the CTA/DMAc system, the partially free draining effect is comparable to the excluded volume effect. It is therefore quite obvious that the dilute solution properties of CTA cannot be described adequately in terms of a simple two-parameters theory.

Accrording to the methods (method $2 \mathrm{C}, 2 \mathrm{E}, 2 \mathrm{~F}$, $2 \mathrm{G}$, and $2 \mathrm{H}),{ }^{4,42}$ the short-range interaction parameter (i.e., the unperturbed chain dimension) $A$ was evaluated for the CTA/DMAc system. Figure 5 represents the Baumann plot (method 2C), Stockmayer-Fixman (SF) plot (method 2E), Kamide et al. plot (method 2F), Kamide-Miyazaki (I) plot (method 2G), and Tanner-Berry plot (method $2 \mathrm{H}$ ). Ail these methods, except method 2E, gave a series of reasonably good straight lines. All plots based on the Stockmayer-Fixman relation showed downward curvature at high molecular weights. The $A$ value and the long-range interaction parameter $B$ (if possible), estimated from the intercepts and slopes of the above plots, are summerized in Table V. The results in Table $\mathrm{V}$ are in particularly good agreement with the theoretical predictions. Methods $2 \mathrm{E}$ and $2 \mathrm{~F}$

\begin{tabular}{|c|c|c|c|c|}
\hline \multirow{2}{*}{ Method } & \multirow{2}{*}{$\frac{A \times 10^{8}}{\mathrm{~cm}}$} & \multirow{2}{*}{$\frac{B \times 10^{27}}{\mathrm{~cm}^{3}}$} & \multirow{2}{*}{$\sigma$} & \multirow{2}{*}{$C_{\infty}$} \\
\hline & & & & \\
\hline 2B $\left(\alpha_{\mathrm{s}}\right.$ from $\left.\psi\right)$ & 1.43 & - & 3.07 & 19.4 \\
\hline $2 \mathrm{C}\left(a_{2}=0\right)$ & 1.49 & 3.9 & 3.20 & 21.1 \\
\hline $2 \mathrm{E}\left(a_{2}=a_{\Phi}=0\right)$ & 0.887 & 4.3 & 1.91 & 7.49 \\
\hline $2 \mathrm{~F}\left(a_{2}=a_{\Phi}=0\right)$ & 0.995 & - & 2.14 & 9.43 \\
\hline $2 \mathrm{G}\left(a_{\Phi} \neq 0\right)$ & 1.47 & 3.7 & 3.16 & 20.6 \\
\hline $2 \mathrm{H}\left(a_{1}=a_{2}=0, a_{\Phi} \neq 0\right)$ & 1.59 & - & 3.42 & 24.1 \\
\hline Most probable value & 1.46 & - & 3.14 & 20.4 \\
\hline
\end{tabular}
considerably understimate the $A$ value due to $a_{\Phi} \neq 0^{42}$ and method $2 \mathrm{H}$, on the contrary, overestimates the $A$ value because $a_{1}+1.5 a_{2}>0 .{ }^{46}$ Three methods $(2 \mathrm{E}, 2 \mathrm{~F}$, and $2 \mathrm{H})$ relaying upon viscosity

Table V. Unperturbed chain dimensions $A$, long-range interaction parameter $B$, conformation parameter $\sigma$, and characteristic ratios $C_{\infty}$ of cellulose triacetate in dimethylacetamide at $25^{\circ} \mathrm{C}$ 
data are unlikely to provide values that are accurate in an absolute sense. As pointed out previously, ${ }^{4,42}$ the SF plot, observed to hold almost universally for vinyl-type polymers, does not hold for cellulose derivatives.

The method $2 \mathrm{~B}, 2 \mathrm{C}$, and $2 \mathrm{G}$ are the most reliable and promising in this respect. In fact, the $A$ values determined by these three methods, within the limits of error are almost the same. Thus as the most probable value, we estimated a $A$ of $1.46 \times 10^{-8} \mathrm{~cm}$.

The conformation parameter $\sigma$, defined by

$$
\sigma=\left\langle S^{2}\right\rangle_{0}^{1 / 2} /\left\langle S^{2}\right\rangle_{0,}^{1 / 2}=A / A_{f}
$$

and the characteristic ratio $C^{\infty}$

$$
C_{\infty}=A_{\infty}{ }^{2} M_{\mathrm{b}} / l^{2}
$$

were calculated subsequently from the $A$ value and tabulated in Table $\mathrm{V}$, where $\left\langle S^{2}\right\rangle_{0}^{1 / 2}$ $\left(=6^{-1 / 2} \times A_{f} M^{1 / 2}\right)$, the rootmean square radius of gyration of a hypothetical chain with free internal rotation, $A_{f}=0.465 \times 10^{-8}$ for CTA and $0.481 \times 10^{-8}$ for CDA; $A_{\infty}$, the asymptotic value of $A$ at infinite molecular weight, $M_{\mathrm{b}}$, mean molecular weight per skeletal bond and $l$, mean bond length. The most probable $\sigma$ and $C_{\infty}$ values are found to be 3.14 and 20.4, respectively for the CTA/DMAc system at $25^{\circ} \mathrm{C}$. These values should be compared with $\sigma=3.59\left(C_{\infty}=26.3\right)$ for CDA in acetone and $\sigma=2.56\left(C_{\infty}=13.5\right)$ for the CDA/THF system. ${ }^{4}$

In order to understand this point better, viscometric and light scattering measurements were made on the solution of a fraction of CDA $(\mathrm{AC}=55.6 \mathrm{wt} \%$, sample code, EF 3-11), prepared by the procedure in the previous paper, ${ }^{4}$ and dissolved in DMAc.

The results are given in Table VI, where the values for $\mathrm{CDA} /$ acetone, $\mathrm{CDA} / \mathrm{THF}$ and CTA/DMAc systems, caclulated from the molecular weight dependence of $[\eta],\left\langle S^{2}\right\rangle_{z}{ }^{1 / 2}, A_{2}$ and $\Phi$, are also shown in parentheses. The results given in the table show that CDA and CTA of the same $M_{w}$ have a very different radius of gyration and limiting viscosity numbers even when determination is made in the same solvent, (DMAc). By use of method 2B, $A=2.27 \times 10^{-8} \mathrm{~cm}$ and $\sigma=4.88$ were evaluated for the CDA/DMAc system. These results indicate that in DMAc, the CTA molecule is much more flexible than the CDA molecule (see Figure 6).

Table $\mathrm{V}$ demonstrates that the $A$ values, estimated by using only viscosity data $\left([\eta]\right.$ and $M_{w}$, or $K_{\mathrm{m}}$ and a), have a large error of \pm about $0.5 \times 10^{-8} \mathrm{~cm}$. Thus, if only the viscosity data are available, we can

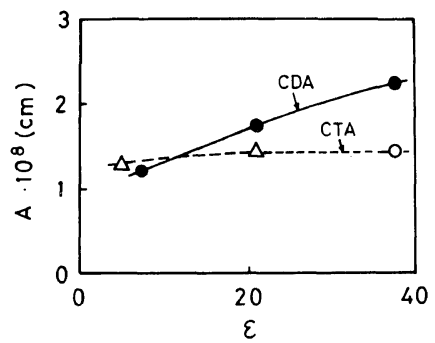

Figure 6. Dependence of the unperturbed chain dimensions $A$ of cellulose triacetate (open mark) and cellulose diacetate (closed mark) on the dielectric constant $\varepsilon$ of the solvents: circle, by method $2 \mathrm{~B}, 2 \mathrm{C}$ (or $2 \mathrm{D}$ ), and $2 \mathrm{G}$; triangle, by method $2 \mathrm{E}$ and $2 \mathrm{~F}$, followed by the correction (see text).

Table VI. Light scattering data on cellulose diacetate in dimethylacetamide(DMAc) as compared with those of cellulose diacetate in acetone and

\begin{tabular}{|c|c|c|c|c|c|c|c|c|c|}
\hline \multirow{2}{*}{ Polymer } & \multirow{2}{*}{ Sample } & \multirow{2}{*}{ Solvent } & \multirow{2}{*}{$\frac{\text { Temp }}{{ }^{\circ} \mathrm{C}}$} & \multirow{2}{*}{$M_{w} \times 10^{-4}$} & \multirow{2}{*}{$\frac{\left\langle S^{2}\right\rangle_{z}^{1 / 2}}{10^{-8} \mathrm{~cm}}$} & \multirow{2}{*}{$\frac{\left\langle S^{2}\right\rangle_{w}{ }^{1 / 2}}{10^{-8} \mathrm{~cm}}$} & \multirow{2}{*}{$\frac{A_{2} \times 10^{4}}{\mathrm{~mol} \mathrm{~cm}{ }^{-3} \mathrm{~g}^{-2}}$} & \multirow{2}{*}{$\frac{[\eta]}{\mathrm{cm}^{3} \mathrm{~g}^{-1}}$} & \multirow{2}{*}{$\Phi \times 10^{-23}$} \\
\hline & & & & & & & & & \\
\hline \multirow{3}{*}{$\begin{array}{l}\text { Cellulose } \\
\text { diacetate } \\
(\mathrm{AC}=55.6 \%)\end{array}$} & \multirow[t]{3}{*}{ EF3-11 } & DMAc & 25 & 10.8 & 344 & 310 & $0.77_{5}$ & 227 & 0.58 \\
\hline & & Acetone & 25 & 10.8 & $(262)^{a}$ & (234) & - & (168) & $(1.00)$ \\
\hline & & THF & 25 & 10.8 & (192) & (173) & - & (149) & $(2.16)$ \\
\hline $\begin{array}{l}\text { Cellulose } \\
\text { triacetate } \\
(\mathrm{AC}=61.0 \%)\end{array}$ & - & DMAc & 25 & 10.8 & (272) & (240) & $(1.25)$ & (157) & $(0.85)$ \\
\hline
\end{tabular}
tetrahydroforan(THF) and of cellulose triacetate in DMAc

a The values in parantheses are calculated from the molecular weight dependence of $\left\langle S^{2}\right\rangle_{z}{ }^{1 / 2}\left(\right.$ or $\left\langle S^{2}\right\rangle_{w}{ }^{1 / 2}$, or $[\eta]$ or $\left.\Phi\right)$ experimentally determined. 
Table VII. Unperturbed chain dimensions $A$ of cellulose triacetate, evaluated by method $2 \mathrm{E}$ and $2 \mathrm{~F}$

\begin{tabular}{|c|c|c|c|c|c|c|}
\hline \multirow{2}{*}{ Method } & \multicolumn{6}{|c|}{$A \times 10^{8} / \mathrm{cm}$} \\
\hline & DMAc $^{\mathrm{a}}$ & $\mathrm{TFA}^{\mathrm{b}}$ & Acetone & $\mathrm{DCM}^{\mathrm{c}}$ & $\mathrm{TCE}^{\mathrm{d}}$ & $\mathrm{TCM}^{\mathrm{e}}$ \\
\hline $2 \mathrm{E}$ & 0.995 & 0.995 & 0.995 & 0.823 & 0.823 & 0.823 \\
\hline $2 \mathrm{~F}$ & 0.995 & 1.00 & 0.995 & 0.853 & 0.876 & 0.884 \\
\hline Most probable value & 1.46 & - & - & - & - & - \\
\hline
\end{tabular}

estimate, by methods $2 \mathrm{E}$ and $2 \mathrm{~F}$, only the comparable variation of the $A$ value with the solvent nature. In Figures $5 \mathrm{~b}$ and $5 \mathrm{c}$ the data points other than those for DMAc are plotted. Table VII shows the $A$ values, estimated by methods $2 \mathrm{E}$ and $2 \mathrm{~F}$, of CTA in various solvents.

From Table VII, it is clear than DMAc, TFA, and acetone give almost the same $A$ values and on the other hand, chlorinated hydrocarbons, like DCM, TCE and TCM, yield slightly smaller $A$ values. Comparison of Tables V and VII thus allow us a rough estimation of the true $A$ value which is found to be $1.46 \times 10^{-8}$ for the former solvents (group I) and $\quad A \simeq 0.87 \times 10^{-8} \times 1.46 / 0.977=1.27 \times 10^{-8}$ (Here, 0.87 is an average value of $A$ estimated by method $2 \mathrm{~F}$ for solutions in solvents of group II (DCM, TCE, and TCM) and 0.977 is a corresponding value for group I (DMAc, TFA, and acetone), respectively) for the latter group of solvents (group II), respectively. Here these values were obtained on the usual assumption that the importance of the draining effect (i.e., $a_{\Phi}$ value) is independent of the solvent nature, but this is not necessarily so. The former is $c a .15 \%$ larger than the latter. The unperturbed chain dimension of CTA, like a variety of other cellulose derivatives differing in chemical composition (for example, cellulose tricaproate and cellulose tricarbanilate), varies with the nature of the solvent. ${ }^{42}$

In Table VIII are compiled the molar volume, dipole moment $\mu$, dielectric constant $\varepsilon$, electric negativity and solubility parameter $\delta$ of the solvents employed in this work. Many of the best solvents for CTA have estimated values of $\delta$ in the range 9-11 $\left(\mathrm{cal} \mathrm{cm}^{-3}\right)^{1 / 2}$. Evidently, all solvents belonging to group I are highly polar (or high dissociable character), capable of displaying a basic or amphoteric nature foward CTA molecules. In contrast to this, the solvents of group II are chlorinated nonpolar hydrocarbons, acting as a weak acids toward CTA

Table VIII. Some physical properties of solvents employed in this study

\begin{tabular}{|c|c|c|c|c|c|c|}
\hline \multirow{2}{*}{ Solvent } & \multirow{2}{*}{$\begin{array}{c}\text { Molar volume } \\
\frac{V_{0}}{\mathrm{~cm}^{3} / \mathrm{mol}}\end{array}$} & \multirow{2}{*}{$\begin{array}{c}\text { Dipole moment } \\
\frac{\mu}{10^{-18} \text { c.u.s }}\end{array}$} & \multirow{2}{*}{$\begin{array}{c}\text { Dielectric } \\
\text { constant } \\
\varepsilon\end{array}$} & \multicolumn{2}{|c|}{$\begin{array}{l}\text { Electric } \\
\text { negativity }\end{array}$} & \multirow{2}{*}{$\begin{array}{l}\begin{array}{l}\text { Solubility } \\
\text { parameter }\end{array} \\
\left(\mathrm{cal} \mathrm{cm}^{-3}\right)^{1 / 2}\end{array}$} \\
\hline & & & & by IR & by NMR & \\
\hline $\mathrm{DMAc}^{\mathrm{a}}$ & 92.3 & $3.79^{47}$ & $37.8^{47}$ & 118 & - & 10.8 \\
\hline $\mathrm{TFA}^{\mathrm{b}}$ & Ca. 100 & $2.30^{48}$ & $39.5^{48}$ & - & - & - \\
\hline Acetone & 73.3 & $\begin{array}{l}2.75^{49} \\
2.90^{48}\end{array}$ & $\begin{array}{l}21.4^{49} \\
20.7^{50}\end{array}$ & 55 & -0.94 & 9.8 \\
\hline $\begin{array}{l}\text { 1-chloro-2,3- } \\
\text { epoxypropane }\end{array}$ & 78.2 & - & $\begin{array}{l}22.6^{48} \\
23^{51}\end{array}$ & - & - & - \\
\hline $\mathrm{DCM}^{\mathrm{c}}$ & 64.1 & $\begin{array}{l}1.89^{49} \\
1.62^{48}\end{array}$ & $\begin{array}{l}7.77^{48} \\
9.08^{50}\end{array}$ & 0 & - & 9.95 \\
\hline $\mathrm{TCE}^{\mathrm{d}}$ & 104.6 & - & $\begin{array}{l}7.29^{47} \\
8.20^{49}\end{array}$ & 0 & - & 9.67 \\
\hline $\mathrm{TCM}^{\mathrm{e}}$ & 80.0 & $1.18^{49}$ & $\begin{array}{l}5.05^{49} \\
4.806^{50}\end{array}$ & 0 & 0 & 9.30 \\
\hline
\end{tabular}

${ }^{a}$ Dimethylacetamide. ${ }^{b}$ Trifluoroacetic acid. ${ }^{c}$ Dichloromethane. ${ }^{d}$ Tetrachloroethane. ${ }^{\mathrm{e}}$ Trichloromethane. 
molecules. This distinct difference in the solvent nature between groups I and II can be considered to be closely related to the significant change in the $A$ value. We have found, in the study of $\mathrm{CDA},{ }^{4}$ that the $A$ value in acetone is larger than that in THF. Apparently, the former solvent has a higher polarity than the latter. Figure 6 shows the plots of the $A$ values of CTA and CDA against the dielectric constants of the solvents, in which the polymers are disolved. Actually, an increase in the dielectric constant invariably accompanies an increase in the $A$ value, particularly for $\mathrm{CDA}$, which is considered to be much more polar than CTA. In other words, both CTA and CDA molecules increase their rigidity as the solvents become polar. Therefore, the polarity of the solvents seems to provide a good key for correlating the $A$ value with the solvent nature. This kind of study is only at its begining stage. Further experimental work is highly desirable before the detailed mechanism, at the molecular level, of the interaction between the solvent and the specific polar group in the cellulosic chain, which is responsible for causing the profound solvent dependence of the short-range interaction parameter, can be well understood.

Moore and Russell ${ }^{37}$ considered that basic solvents (like group I) may interact with hydroxyl group and break the assumed intramolecular hydrogen bonding between the hydroxyl and acetyl groups existing in CDA molecules and the change in $[\eta]$ with the solvent can be interpreted in terms of the intramolecular hydrogen bonding. If Moore and Russell's hypothesis is justified even for not fully substituted CTA (in this study, DS $=2.89$ ), then it follows that group I should give lower $[\eta]$ than group II. The experimental results, showing just the reverse

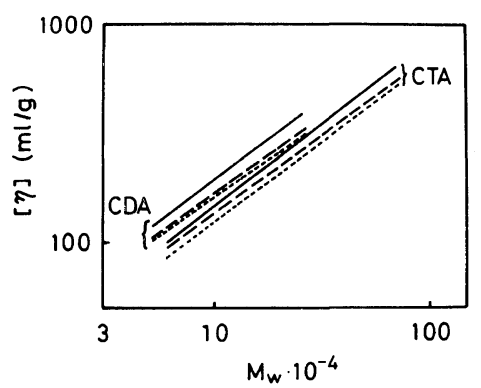

Figure 7. Typical Mark-Houwink-Sakurada relations for cellulose triacetate (CTA) and cellulose diacetate (CDA) in dimethylacetamide (full line), trifluoroacetic acid (broken line), and acetone (dotted line). of this prediction, indicates that this hypothesis is not valid.

As noted before, the $\chi$ value, and accordingly the $B$ value, are almost independent of the solvent nature. However, $[\eta]$ increases in the order: TCM $<$ TCE $<$ acetone $<$ DMAc, since $[\eta]$ depends not only on the solvent power, but also on $A$, the draining effect and the non-gaussian nature. In summary, the effect of the solvent on the dilute solution properties is shown not by $B$ but by $A$. This conclusion is generally accepted for cellulose derivatives.

Effect of the Degree of Substitution of Cellulose Acetate on $[\eta]$ and the Unperturbed Chain Dimension

Table IX summerizes the viscosity data on solutions of five CDA (DS $=2.46$ ) fractions, employed in the previous paper, ${ }^{4}$ in DMAc and TFA at $25^{\circ} \mathrm{C}$. The table includes also the literature data $\left(M_{w}\right.$ by LS and $[\eta]$ ) in acetone. ${ }^{4}$

The following MHS equations were established from these $[\eta]-M_{w}$ data by the least square(s) method for CDA in DMAc and TFA, respectively

$$
[\eta]=3.95 \times 10^{-2} M_{w}{ }^{0.738} \quad \text { in DMAc }
$$

and

$$
[\eta]=5.27 \times 10^{-2} M_{w}^{0.696} \quad \text { in TFA }
$$

We have already obtained the MHS equation for CDA in acetone. ${ }^{4}$

$$
[\eta]=0.133 M_{w}{ }^{0.616} \quad \text { in acetone }
$$

Figure 7 depicts the MHS equations established for CTA and CDA in DMAc, TFA, and acetone by straight lines. As far as DMAc, TFA, and acetone

\begin{tabular}{|c|c|c|c|c|}
\hline \multirow{2}{*}{$\begin{array}{l}\text { Sample } \\
\text { code }\end{array}$} & \multirow{2}{*}{$M_{w} \times 10^{-4^{\mathrm{a}}}$} & \multicolumn{3}{|c|}{$[\eta]$ at $25^{\circ} \mathrm{C} / \mathrm{cm}^{3} \mathrm{~g}^{-1}$} \\
\hline & & $\mathrm{DMAc}^{\mathrm{b}}$ & $\mathrm{TFA}^{\mathrm{c}}$ & Acetone $^{\mathrm{d}}$ \\
\hline EF 2-10 & 6.1 & 140 & 113 & 117 \\
\hline EF 2-11-1 & 9.6 & 170 & 145 & 151 \\
\hline EF 3-10 & 10.6 & 205 & 178 & 160 \\
\hline EF 3-12 & 14.1 & 243 & 193 & 193 \\
\hline EF 3-14 & 18.5 & 315 & 247 & 241 \\
\hline
\end{tabular}

Table IX. Intrinsic viscosity number of cellulose diacetate in various solvents

a By LS in acetone solution. ${ }^{\mathrm{b}}$ Dimethylacetamide.

c Trifluoroacetic acid. ${ }^{\mathrm{d}}$ Ref 4. 
Table X. $\quad[\eta], \Phi, A$, and $\alpha_{\mathrm{s}}$ data for cellulose triacetate(CTA) and cellulose diacetate(CDA) as compared with polychloroprene(PCP) and poly $\left(\alpha\right.$-methylstyrene) $\left(\mathrm{P} \alpha\right.$-MS) for $M_{w}=1 \times 10^{5}$

\begin{tabular}{|c|c|c|c|c|}
\hline \multirow{2}{*}{ Polymer/Solvent } & {$[\eta]$} & \multirow{2}{*}{$\Phi \times 10^{-23}$} & $A_{(\mathrm{m})} \times 10^{8}$ & \multirow{2}{*}{$\alpha_{\mathrm{s}}$} \\
\hline & $\mathrm{cm}^{3} \mathrm{~g}^{-1}$ & & $\mathrm{~cm}$ & \\
\hline CTA/DMAc ${ }^{a}$ & $148^{d}(156)^{e}$ & 1.19 & 1.46 & 1.10 \\
\hline CDA/acetone & $160 \quad(156)$ & 0.90 & 1.73 & 1.02 \\
\hline $\mathrm{CDA} / \mathrm{THF}^{\mathrm{b}}$ & $141 \quad(141)$ & 1.97 & 1.23 & 1.07 \\
\hline $\mathrm{CDA} / \mathrm{DMAc}$ & $193(-)$ & 0.58 & 2.27 & 1.02 \\
\hline $\mathrm{PCP} / \mathrm{MEK}^{\mathrm{c}}$ & $35(35)$ & 2.9 & 0.72 & 1.0 \\
\hline $\mathrm{P}(\alpha-\mathrm{MS}) / t$-decaline $\mathrm{e}^{54.55}$ & $21(21)$ & 2.3 & 0.67 & 1.0 \\
\hline $\mathrm{P}(\alpha-\mathrm{MS}) /$ cyclohexane $^{56,57}$ & $23(26)$ & 2.3 & 0.71 & 1.0 \\
\hline
\end{tabular}

${ }^{a}$ Dimethyl acetamide. ${ }^{b}$ Tetrahydrofuran. ${ }^{\mathrm{c}}$ Methyl ethyl ketone. ${ }^{\mathrm{d}}$ Calculated from MHS equations. ${ }^{\mathrm{e}}$ Calculated by eq 18 from $\Phi, A$, and $\alpha_{\mathrm{s}}$ data.

are concerned, in a given solvent $[\eta]$ is about 20 $30 \%$ smaller for CTA than it is for CDA of the same $M_{w}$.

The limiting viscosity number $[\eta]$ can be calculated from $\Phi, A, M_{w}$, and $\alpha_{s}$ through use of the well-known Flory-Fox equation:

$$
[\eta]=\Phi A^{3} M_{w^{1}}{ }^{1 / 2} \alpha_{\mathrm{s}}{ }^{3}
$$

In Table $\mathrm{X}$ are listed the values of [ $\eta], \Phi, A, \alpha_{\mathrm{s}}$ for CTA and CDA with $M_{n}=1 \times 10^{5}$. In this case, $[\eta], \Phi$ and $\alpha_{s}$ are interpolated from their molecular weight dependence experimentally determined and can be approximately considered as the experimental values. The parenthesis in the table means the $[\eta]$ value calculated from eq 21 . The calculated values are roughly in agreement with the experimental ones. Small $\Phi$, small $\alpha_{\mathrm{s}}$ near to unity, and large $A$ are the features of cellulose acetate solutions. ${ }^{42}$ For the sake of comparison, the corresponding data ${ }^{42}$ obtained for polychloroprene, ${ }^{54,55}$ and $\operatorname{poly}(\alpha-$ methylstyrene $)^{56,57}$ in the solvents are included in Table X. The experimental results indicating that the $[\eta]$ value of cellulose acetate (and probably other cellulose derivatives) solutions is about 3 to 10 times greater than that of the flexible, less polar polymer of an equivalent molecular weight, may be clearly ascribed to the large unperturbed chain dimensions due to the rigidity of the cellulosic chains.

Figure 8 shows the effect of the degree of substitution (DS) of cellulose acetate and cellulose nitrate $(\mathrm{CN})$ with $M_{w}=1 \times 10^{5}$ on their $[\eta]$ and $\sigma$ in acetone and DMAc. Here the data points of $\mathrm{CN}$ are the results in the previous paper, ${ }^{42}$ analyzed from

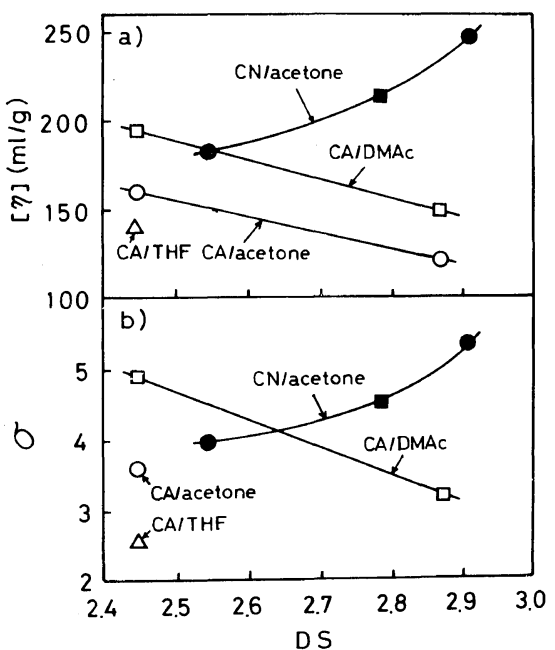

Figure 8. Effect of the degree of substitution (DS) on the limiting viscosity number $[\eta]$ of cellulose acetate (CA) and cellulose nitrate (NC) with $M_{w}=1 \times 10^{5}$ in acetone, dimethylacetamide (DMAc) and tetrahydrofuran (THF) and their conformation parameter $\sigma: \square, \mathrm{CA}$ in DMAc (this work); $\bigcirc, \mathrm{CA}$ in acetone (this work and Kamide et al.'s work ${ }^{4}$ ); $\triangle$, CA in THF (Kamide et al.'s work ${ }^{4}$ ); $\mathrm{CN}$ in acetone (data from Schulz and Penzel's work ${ }^{58.59}$ ); $\square, \mathrm{CN}$ in acetone (data by Huque et al. ${ }^{60}$ ).

works by Schulz and Penzel, ${ }^{58,59}$ and by Huque et $a l^{60}[\eta]$ as well as $\sigma$ increases with DS in $\mathrm{CN}$ solutions, but decreases in CA solutions. A Similar tendency for $[\eta]$ were observed by Howlette et al. ${ }^{61}$, Moore and Russel, ${ }^{62,63}$ and $\mathrm{Ueda}^{64}$ for CA and Warrow, ${ }^{65}$ and Linsley and Frank ${ }^{66}$ for $\mathrm{CN}$. These truly remarkable differences between $\mathrm{CA}$ and $\mathrm{CN}$, 
with respect to the effect of DS, may be attributable to the fact that the CA molecule acts as proton acceptor to the solvents in question and the $\mathrm{CN}$ molecules act as electron acceptors.

Acknowledgement. The authors wish to express their gratitude to Dr. Kunihiko Okajima of this laboratory and to Mr. Hiroyuki Makino of Tokyo Institute of Technology for their fruitful discussion and technical assistance.

\section{REFERENCES}

1. See, for example, W. Brown, "Cellulose Derivatives," N. M. Bikales and L. Segal, Ed., Wiley, New York, N.Y., 1971, Chapter XIV, G, p 589.

2. M. Kurata, Y. Tsumashima, M. Iwata, and K. Kamata in "Polymer Handbook," 2nd ed, J. Brandrup and E. H. Immergut, Ed., John Wiley \& Sons, New York, N.Y., 1975.

3. P. R. M. Nair, R. M. Gohil, K. C. Patel, and R. D Patel, Eur. Polym. J., 13, 273 (1977).

4. K. Kamide, T. Terakawa, and Y. Miyazaki, Polym. $J$, in press.

5. See, for example, K. Sobue, "Cellulose Handbook," Asakura, Tokyo, 1948.

6. K. Kamide, S. Manabe, and E. Osafune, Makromol. Chem., 168, 173 (1973).

7. H. Lachs, J. Kronman, and J. Wajs, Kolloid Z., 79, 91 (1937).

8. G. R. Levi and A. Giera, Gazz. Chim. Ital., 67, 719 (1937).

9. G. R. Levi, U. Villota, and M. Montirelli, Gazz. Chim Ital., 68, 589 (1938).

10. S. Bezzi and U. Croatta, Atti Inst. Veneto Sci., 99, 905 (1939-1940).

11. A. Munster, J. Polym. Sci., 5, 333 (1950).

12. H. Sobue, K. Matsuzaki, and K. Yamakawa, Sen'i Gakkaishi, 12, 100 (1956).

13. G. Langhammer, Makromol. Chem., 24, 74 (1956).

14. R. J. E. Cumberbrich and W. G. Harland, J. Text, Inst., 28, T679 (1958).

15. K. Thirius and L. Dimter, Plaste Kautsch., 6, 547 (1959).

16. I. Kido and K. Suzuki, Sen'i Gakkaishi, 16, 83 (1960).

17. P. A. Okunev and O. G. Terakanov, Khim. Volokna, 6, 44 (1963).

18. N. P. Dymarchuk, K. P. Mischenko, and T. V. Formina, Zh. Prikl. Khim. (Leningrad), 37, 2263 (1964).

19. P. Howard and R. S. Parikh, J. Polym. Sci., A-1, 4, 407 (1966).

20. K. H. Bischoff and B. Phillipp, Faserforsch. Textiltech., 17, 395 (1966).

21. J. M. G. Cowie and R. S. Ranson, Makromol. Chem., 143, 105 (1971).
22. See, for example, K. Kamide, "Fractionation of Synthetic Polymers," L. H. Tung Ed., Marcel Dekker Inc., New York, N.Y., 1977, Chapter 2.

23. K. Kamide, Y. Miyazaki, and T. Abe, Polym. Prepr., Jpn., 26, 1164 (1977).

24. C. L. Smart and C. N. Zellner, "Cellulose and Cellulose Derivatives," N. M. Bikales and L. Segal, Ed., Wiley, New York, N.Y., 1971, Chapter XIX, C, p 1151.

25. G. Kita, I. Sakurada, and T. Nakajima, Kogyo Kagaku Zasshi, 30, 484 (1927).

26. K. Kamide and Y. Miyazaki, Makromol. Chem., 176, 1029 (1975).

27. K. Kamide and Y. Miyazaki, Makromol. Chem., 176, 1051 (1975).

28. K. Kamide and Y. Miyazaki, Makromol. Chem., 176, 1427 (1975).

29. K. Kamide and Y. Miyazaki, Makromol. Chem., 176, 1447 (1975)

30. K. Kamide, K. Sugamiya, T. Ogawa, C. Nakayama, and N. Baba, Makromol. Chem., 135, 23 (1970).

31. K. Kamide, K. Sugamiya, T. Terakawa and T. Hara, Makromol. Chem., 156, 287 (1972).

32. K. Kamide, Y. Miyazaki, and T. Abe, Makromol. Chem., 177, 485 (1976).

33. K. Kamide, Y. Miyazaki, and T. Abe, Polym. J., 9, 395 (1977).

34. I. Noda, K. Kamide, Y. Miyazaki, and H. Ishikawa, Polym. Prepr., Jpn., 27, 559 (1978).

35. H. Staudinger and T. Eicher, Makromol. Chem., 10, 261 (1953).

36. See, for example, W. R. Moore, and J. Russell, J. Colloid Sci., 9, 338 (1954).

37. K. Kamide, Y. Miyazaki, and T. Abe, to be published.

38. P. J. Flory, O. K. Spurr, Jr., and D. K. Carpenter, J. Polym. Sci., 27, 231 (1958).

39. H. J. Phillipp and C. F. Bjork, J. Polym. Sci., 6, 549 (1951).

40. A. Sharples and H. M. Major, J. Polym. Sci., 27, 433 (1958).

41. M. I. Shakhparonov, N. P. Zahurdayeva, and Ye. K. Podgarodetskii, Vysokomol. Soedin., Ser. A, 9, 1212 (1967).

42. K. Kamide and Y. Miyazaki, Polym. J., 10, 409 (1978).

43. - E. F. Casassa, Polymer, 3, 625 (1962).

44. M. Murata and H. Yamakawa, J. Chem. Phys., 29, 311 (1958).

45. M. Kurata, "Modern Industrial Chemistry," Vol. 18, Polymer Industrial Chemistry III, Asakura, Tokyo, 1975, p 287.

46. K. Kamide and T. Terakawa, Polym. J., 10, 559 (1978).

47. R. Oda, N. Tokura, and Z. Yoshida, Ed., "New Organic Synthesis Using Polar Solvents": Chemistry," Supplment No. 52, (in Japanese) Kagaku Dojin, Kyoto, 1971.

48. The Chemical Society of Japan, Ed., "Chemistry 
Handbook," Fundamental Part II, 2nd ed, Maruzen, Tokyo, 1975.

49. J. A. Riddick, "Organic Solvents," 2nd ed, Interscience Publishers, New York, N.Y., 1955.

50. N. A. Range, Ed., "Handbook of Chemistry," 10th ed, McGraw-Hill, New York N.Y., 1967.

51. C. Marsden and S. Mann, "Solvent Guide," CleaverHume Press, London, 1963.

52. C. Agami and M. Caillot, Bull. Soc. Chim. Fr., 1990 (1969).

53. J. D. Crowley, G. S. Teague, and J. W. Lower, J. Paint Technol., 38, 269 (1966).

54. T. Norisuye, K. Kuwahara, A. Teramoto, and H. Fujita, J. Chem. Phys., 49, 330 (1968).

55. K. Kuwahara, T. Norisuye, and H. Fujita, J. Chem. Phys., 49, 4339 (1968).

56. T. Kato, K. Miyaso, I. Noda, T. Fujimoto, and M. Nagasawa, Macromolecules, 3, 777 (1970).
57. U. Noda, K. Mizutani, T. Kato, T. Fujimoto, and M. Nagasawa, Macromolecules, 3, 787 (1970).

58. G. V. Schulz and E. Penzel, Makromol. Chem., 112, 260 (1968).

59. E. Penzel and G. V. Schulz, Makromol. Chem., 113, 64 (1968).

60. M. M. Huque, D. A. Goring, and S. G. Mason, Can. J. Chem., 36, 952 (1958).

61. F. Howlette, E. Minshall, and A. R. Urquhart, Shirley Inst. Mem., 18, 251 (1941).

62. W. R. Moore and J. Russell, J. Colloid Sci., 8, 243 (1953).

63. W. R. Moore and J. Russell, J. Colloid Sci., 9, 4 (1954).

64. K. Uda, Sen'i Gakkaishi, 18, 105 (1962).

65. H. A. Warrow, Kolloid Z., 102, 29 (1943).

66. C. H. Linsley and M. B. Frank, Ind. Eng. Chem., 45, 2491 (1953). 